\title{
Combined oxidative phosphorylation defect type 8
}

INSERM

\section{Source}

INSERM. (1999). Orphanet: an online rare disease and orphan drug data base. Combined oxidative phosphorylation defect type 8. ORPHA:319504

Combined oxidative phosphorylation defect type 8 is a mitochondrial disease due to a defect in mitochondrial protein synthesis resulting in deficiency of respiratory chain complexes I, III and IV in the cardiac and skeletal muscle and brain characterized by severe hypertrophic cardiomyopathy, pulmonary hypoplasia, generalized muscle weakness and neurological involvement. 